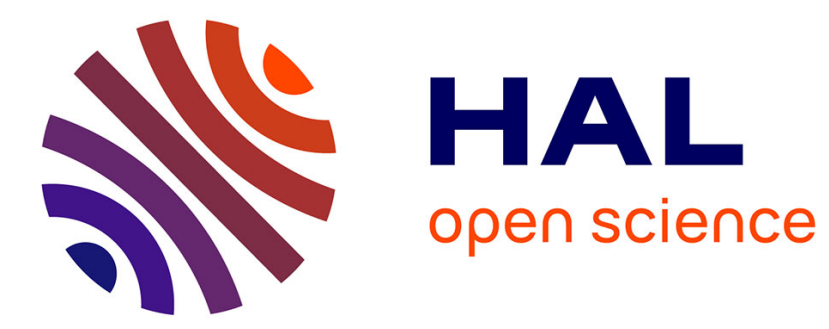

\title{
Mirage technique in anisotropic solids
}

Xavier Quélin, Bernard Perrin, G. Louis

\section{To cite this version:}

Xavier Quélin, Bernard Perrin, G. Louis. Mirage technique in anisotropic solids. Journal de Physique

IV Proceedings, 1994, 04 (C7), pp.C7-275-C7-278. 10.1051/jp4:1994765 . jpa-00253294

\section{HAL Id: jpa-00253294 \\ https://hal.science/jpa-00253294}

Submitted on 1 Jan 1994

HAL is a multi-disciplinary open access archive for the deposit and dissemination of scientific research documents, whether they are published or not. The documents may come from teaching and research institutions in France or abroad, or from public or private research centers.
L'archive ouverte pluridisciplinaire HAL, est destinée au dépôt et à la diffusion de documents scientifiques de niveau recherche, publiés ou non, émanant des établissements d'enseignement et de recherche français ou étrangers, des laboratoires publics ou privés. 


\title{
Mirage technique in anisotropic solids
}

\author{
X. Quelin, B. Perrin and G. Louis \\ Université Pierre et Marie Curie Paris VI, Département de Recherches Physiques, URA 71 du CNRS, T22, \\ Boîte 136, 4 place Jussieu, 75252 Paris cedex 05, France
}

\begin{abstract}
Theoretical and experimental analysis of heat diffusion in an anisotropic medium are presented. The solution of the 3D thermal conduction equation in an orthorhombic medium is calculated by the mean of a Fourier transforms method. Experiments were performed on an orthorhombic polydiacetylene single crystal sample. The temperature field at the sample surface was determined using the photothermal probe beam deflection technique. Then the 3 coefficients of the thermal conductivity tensor have been measured.
\end{abstract}

\section{INTRODUCTION}

Many theoretical models for thermal diffusion of heat have been reported $[1,2]$, but only a few experimental investigations concerning anisotropic media are available. We can point out the flash method [3] and the "steady-state potentiometric" technique [4] which have been used to analyse thermal properties of orthotropic polymer samples.

For our study, the experimental device used is the photothermal probe beam deflection technique [5, 6]. This method requires no mechanical contact with the sample and thus experiments on very small samples can be performed.

We present a numerical simulation of the probe beam deflection for pure thermal waves progressing in an orthorhombic sample, using the Fourier transform of the generalized 3D thermal conduction equation.

The sample under study is a polydiacetylene single crystal of poly-4BCMU (BCMU for butoxycarbonylmethylurethane). This orthorhombic crystal of conjugated unbranched polymer chains extending in the crystallographic $\mathbf{c}$ direction [7], is in a (b,c) planar configuration.

We have determined the $3 \mathrm{D}$ thermal conductivity tensor of this sample from the analysis of the tangential component of the deflection angle, versus the offset between the pump and the probe beams.

\section{THEORY}

\subsection{Basic analysis}

In the case of an orthorhombic geometry, the second order conductivity tensor can be written as

$$
\overline{\bar{\kappa}}=\left(\begin{array}{ccc}
\kappa_{c} & 0 & 0 \\
0 & \kappa_{b} & 0 \\
0 & 0 & \kappa_{a}
\end{array}\right)
$$

where $\kappa_{\mathrm{c}}, \kappa_{\mathrm{b}}$ denote the thermal conductivity coefficients respectively parallel and perpendicular to the chain direction, and $\kappa_{\mathrm{a}}$ perpendicular to the $(\mathbf{b}, \mathbf{c})$ plane. Then, according to the energy conservation law, the stationary $3 \mathrm{D}$ thermal conduction equation in such a medium, in the reference frame relative to the crystallographic axis (Fig.1) and for a modulation frequency $f=\omega / 2 \pi$ is : 


$$
\overline{\bar{\kappa}} \nabla^{2} \tau_{s}(u, v, z)+i \rho C_{p} \omega \tau_{s}(u, v, z)=-\frac{I_{0}}{2 \pi r_{g}^{2}} \exp \left(-\frac{u^{2}+v^{2}}{2 r_{g}^{2}}\right) \exp (\beta z)
$$

where $I_{0}$ and $r_{g}$ are respectively the power and the radius of the pump beam. The density, the specific heat and the optical-absorption-rate constant of the sample are denoted by $\rho, C_{p}$ and $\beta$. The right hand term describes the heat source which keeps the cylindrical symmetry of the gaussian pump beam profile; this term is equal to zero in the thermal conduction equation of the surrounding media (respectively $\tau_{g}$ and $\tau_{b}$ for the front and backing air).

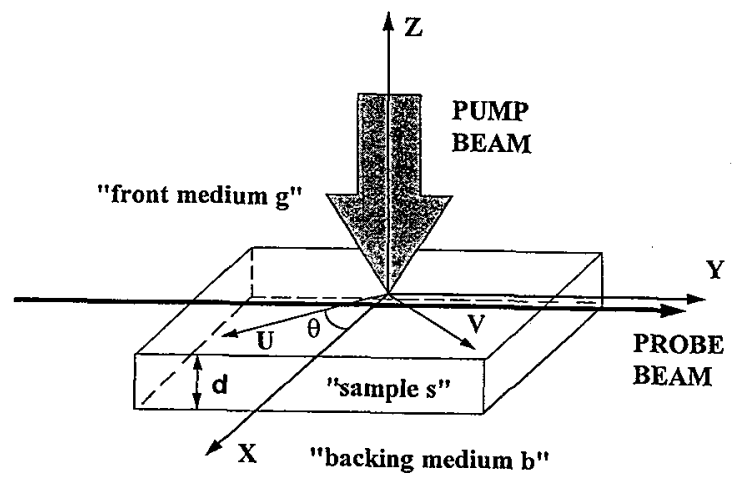

FIG. 1. Schematic description of the geometrical configuration. The experimental device is attached to the $(X, Y, Z)$ axis, whereas the $(U, V, Z)$ frame refers to the crystallographic $(c, b, a)$ axes (where $c$ denotes the chain direction). The angle $(c, X)$ is denoted by $\theta$.

In order to obtain the temperature distribution within the sample, we used a $2 \mathrm{D}$ Fourier transform in the $(u, v)$ plane, taking account of the boundary conditions at the interfaces (continuity of temperature and heat flow) between the sample and the air. We can consider the sample as infinite in the (u,v) plane, because of its dimensions larger than the caracteristic thermal wavelength associated to our frequency modulation range. The temperature distribution $\tau_{\mathrm{g}}$ in the probed atmosphere is deduced from the integral in Fourier space :

$$
\tau_{g}(u, v, z)=\iint_{-\infty}^{+\infty} d k_{u} d k_{v} \exp \left[-i\left(k_{u} u+k_{v} v\right)\right] \exp \left[-\sigma_{g}\left(k_{u}, k_{v}\right) z\right] T_{g}\left(k_{u}, k_{v}\right)
$$

where $\mathrm{T}_{\mathbf{g}}(\mathbf{k})$ is the temperature in the Fourier space [8] and $\sigma_{g}^{2}=k_{u}^{2}+k_{v}^{2}+2 i l_{g}^{-2}$. In this formula, $l_{g}=\left(2 \kappa_{g} / \rho_{g} C_{g} \omega\right)^{1 / 2}$ denotes the thermal diffusivity length in the probed air.

The probe beam deflection $\phi$ is given by the line integral of the temperature gradient along the whole optical path L :

$$
\phi=-\frac{1}{n} \frac{d n}{d T} \int_{L} \nabla \tau_{g} \times d l .
$$

Then, with the appropriate expression of $\tau_{\mathrm{g}}$ in the $(X, Y, Z)$ reference frame, the tangential component $\phi_{t}$ is :

$$
\phi_{t} \propto \int_{-\infty}^{+\infty} \frac{\partial \tau_{g}}{\partial x} d y, \text { which reduces to }: \phi_{t} \propto \int_{-\infty}^{+\infty} d k k \sin (k x) T_{g}(k) \exp \left[-\sigma_{g}(k) z\right]
$$

where $\sigma_{g}^{2}(k)=k^{2}+2 i l_{g}^{-2}$. Equation (1) is numerically calculated to compare our theoretical model with experimental data.

\subsection{Asymptotic derivation}

Physical considerations lead to simplifications in the theory presented above : 
in our case, the high value of the optical absorption constant $\left(\beta=2 \times 10^{5} \mathrm{~cm}^{-1}\right.$ compared to a sample thickness $\mathrm{d}=70 \mu \mathrm{m})$, and the existence of a mismatch between the densities of the sample $\left(\rho_{\mathrm{s}}\right)$ and the probed medium $\left(\rho_{\mathrm{g}}\right), \quad\left(\rho_{g}\left\langle\left\langle\rho_{s}\right)\right.\right.$, lead to the following assumptions : $\exp (-\beta d) \approx 0$, and $\sigma_{g} \kappa_{g} / \sigma_{s} \kappa_{a} \approx \sigma_{b} \kappa_{b} / \sigma_{s} \kappa_{a} \approx 0$;

we introduce an effective thermal conductivity coefficient in the $(\mathbf{b}, \mathbf{c})$ plane, relative to the scanning direction $\mathrm{x}$ :

$$
\kappa_{\theta}=\kappa_{c} \cos ^{2} \theta+\kappa_{b} \sin ^{2} \theta
$$

and a rescaled sample thickness $\tilde{d}=d \sqrt{\kappa_{\theta} / \kappa_{a}}$, equation (1) becomes :

$$
\phi_{t}=-\frac{1}{n} \frac{d n}{d T} \frac{I_{0}}{\sqrt{\kappa_{a} \kappa_{\theta}}} \int_{0}^{+\infty} d k k \sin (k x) \exp \left[\frac{-r_{g}^{2} k^{2}}{8}\right] \frac{1}{\sigma_{\theta}} \frac{1+\exp \left(-2 \sigma_{\theta} \tilde{d}\right)}{1-\exp \left(-2 \sigma_{\theta} \tilde{d}\right)} \exp \left(-\sigma_{g} z\right)
$$

where $\sigma_{\theta}^{2}=k^{2}+2 i l_{\theta}^{-2}$, and $l_{\theta}=\sqrt{2 \kappa_{\theta} / \rho_{s} C_{s} \omega}$ denotes the thermal diffusion length in the direction $\theta$. Under such conditions, equation (2) has the same form as in an isotropic medium [9].

Furthermore, in the asymptotic limits of a thermally thin $\left(d<<l_{s}\right)$, and of thermally thick $\left(d \gg l_{s}\right)$ samples, the integrand is proportional to $\sigma_{\theta}^{-2}$ and $\sigma_{\theta}^{-1}$, and independant of $\kappa_{\mathrm{a}}$. So, for low frequency experiments, equation (3) reduces to:

$$
\phi_{t}(x, z) \propto \int_{0}^{+\infty} d k k \sin (k x) \exp \left(\frac{-r_{g}^{2} k^{2}}{8}\right) \frac{1}{\sigma_{\theta}^{2}} \exp \left(-\sigma_{g} z\right)
$$

Under these conditions, it can be schown [8] that the value of $\kappa_{\theta}$ can be derived from the dependence of the amplitude $A_{t}$ of $\phi_{t}$ in terms of the transverse off set $x$ (for large values of $x$ ).

\section{EXPERIMENTS}

Experiments were performed on a conventional mirage detection setup, which is described in detail in ref. [8]. Its summarized main features are the following: a $8 \mathrm{~mW}$-power argon laser beam, tuned on 488 $\mathrm{nm}$ wavelength, provides the polarized gaussian profile pump beam. The scanning of the distance $X_{0}$ between the pump and the probe beams is monitored by a $0.1 \mu \mathrm{m}$ precision step motor. The low-frequency modulation $(0.1-20 \mathrm{~Hz})$ is accomplished by an electromechanical shutter (nm Laser Products LS 200).

Monocrystals of poly-4BCMU [10] are obtained by irradiating monomer single crystals with $\gamma$ rays. These monocrystals are then cleaved along the (b,c) plane and typically 50 to $100 \mu \mathrm{m}$ thick, $10 \mathrm{~mm}$ long and $6 \mathrm{~mm}$ wide samples were available. These samples were hold by their edges on a Pyrex substrate, with an air layer between the sample and Pyrex holder. The chain direction was found from the strong optical dichroism of the 4BCMU. The maximum absorption of light accurs when the polarization of incident light is parallel to $\mathbf{c}$.

\section{RESULTS}

Experiments have been performed on samples of differents thickness. As the results were the same within experimental error, we present data for only one sample of $70 \mu \mathrm{m}$ thickness. In Fig. 2 we have plotted the experimental data for $\theta=90^{\circ}$ (which means that $X_{0}$ is progressing in the perpendicular chain's direction). The curves exhibit a maximum which is closely related to the pump beam radius $\mathrm{r}_{\mathrm{g}}$ but does not influence the plot for large values of $X_{0}$. For the low frequency experiments $(0.5$ and $1 \mathrm{~Hz})$, the slopes of the lines give $\kappa_{b}=0.42 \mathrm{~W} \mathrm{~m}^{-1} \mathrm{~K}^{-1}$. The different behavior of the high-frequency experiments (4 and 10 $\mathrm{Hz}$ ) allows us to pinpoint the crossover between the thermally thin and thick regimes. A numerical analysis of these higher-frequency measurements, according to Eq.(1), leads to an estimation of $\kappa_{\mathrm{a}}=0.025 \mathrm{~W} \mathrm{~m}^{-1}$ $\mathrm{K}^{-1}$. Computed lines show quite good agreement with experiments.

In the same way, a value of $\kappa_{\mathrm{c}}=2.2 \mathrm{~W} \mathrm{~m}^{-1} \mathrm{~K}^{-1}$ can be deduced from low frequency experiments with $\theta=0$. For intermediate directions, different values of $\kappa_{\theta}$ derived from the slope of low-frequency 
experiments have been found in good agreement with the theoretical dependence of $\kappa_{\theta}$ with $\theta$, according to Eq.(2) with $\kappa_{c}$ and $\kappa_{b}$ previously determined.

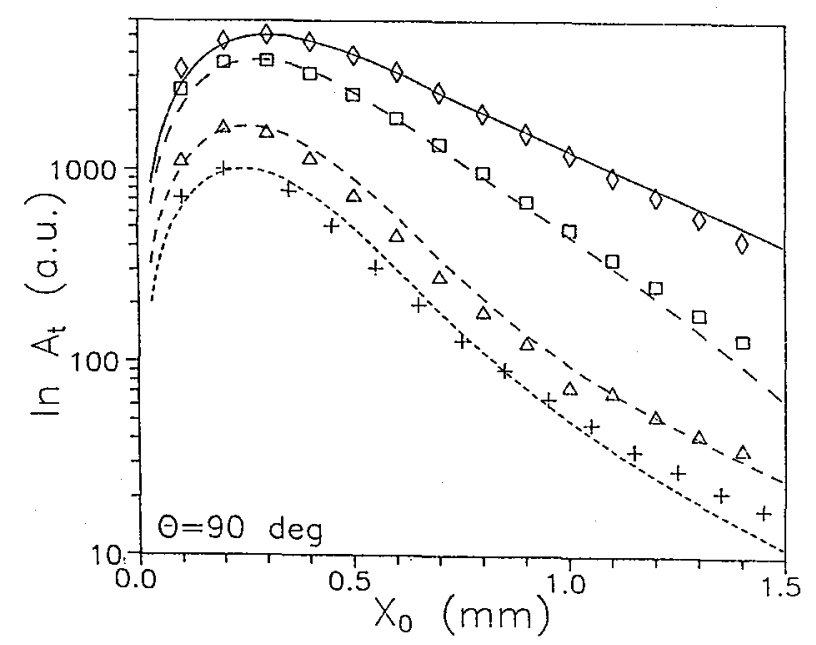

FIG. 1. Logarithm of the amplitude $\left(A_{t}\right)$ of $\phi_{t}\left(\right.$ for $\left.\theta=90^{\circ}\right)$, is plotted for different modulation frequencies (symbols represent experimental data). According to Eq.(1), solid lines were computed for each frequency with the values $r_{g}=360 \mu \mathrm{m}, d=70 \mu \mathrm{m}, z=150 \mu \mathrm{m}, \rho_{s}=1170 \mathrm{~kg} \mathrm{~m}^{-3}, C_{s}=1480 \mathrm{~J} \mathrm{~kg}^{-1}$ $K^{-1}, \kappa_{b}=0.42 \mathrm{~W} \mathrm{~m}^{-1} \mathrm{~K}^{-1}$ and $\kappa_{a}=0.025 \mathrm{~W} \mathrm{~m}^{-1} \mathrm{~K}^{-1}$.

\section{CONCLUSION}

We have shown that photothermal-probe-deflection is a suitable technique to study anisotropic thermal conductivity behavior of materials, even when only small samples are available, due to the contactless character of this method. The 3D thermal diffusion equation of heat in an orthorhombic medium was solved using a 2D Fourier transform. So the thermal field in the probed front air can be numerically computed.

The thermal conductivity tensor has been measured by the analysis of the transverse component of the probe beam deflection. We have showed that for optically thick and thermally thin samples, the complete expression reduces to the same one as in an isotropic medium, provided that an effective thermal conductivity relative to the chain direction and a rescaled sample thickness are defined. Moreover, physical considerations lead to simplifications in the theory so that direct measurements of the thermal conductivity were performed.

Various effects may be invoked to explain the large anisotropic behavior of 4BCMU (presence of hydrogens bonds along the chains axis, lamellar structure), but further experimental data (sound velocities, temperature dependence measurements) would be necessary for a complete heat diffusion analysis.

\section{References}

[1] Carslaw H.S. and Jaeger J.C., Conduction of heat in solids (Oxford University Press, Oxford, 1959).

[2] Vaez Iravani M. and Nikoonahad M., J. Appl. Phys. 62 (1987) 4065-4071.

[3] Choy C.L., Luk W.H. and Chen F.C., Polymer 19 (1978) 155-162.

[4] Murray C.M., Wybourne M.N., Batchelder D.N. and Ade P.A.R., J. Phys. Condens. Matter 2 (1990) 257-264.

[5] Boccara A.C., Fournier D. and Badoz J., Appl. Phys. Lett. 36 (1980) 130-132.

[6] Zhang X., Changming G., Zhang Q., Hong Y. and Peizhen F., in this issue.

[7] Brouty C., Spinat P. and Whuler A., Acta. Crystallogr. C 44 (1988) 1070-1076.

[8] Quelin X., Perrin B., Louis G. and Peretti P., Phys. Rev. B 48 (1993) 3677-3682.

[9] Salazar A., Sanchez-Lavega A. and Fernandez J., J. Appl. Phys. 69 (1991) 1216-1223.

[10] samples provided by J. Berrehar, C. Lapersonne-Meyer and M. Schott, GPS, Univers. Paris 7 and 6. 